\title{
Die Replantation
}

\section{Nico Leibig, Ulrich Kneser, Berthold Bickert}

\section{Zusammenfassung}

Amputationsverletzungen sind insgesamt eher seltene, für den einzelnen Betroffenen aber schwerwiegende Verletzungen, welche $\mathrm{zu}$ erheblichen Einschränkungen der Berufsfähigkeit und der freizeitlichen Aktivitäten führen können. Darüber hinaus entsteht ein deutlich sichtbarer körperlicher Schaden, welcher mit dem Verlust des Gefühls der Unversehrtheit einhergeht. Bereits im Vorfeld der Operation kann meist anhand des Patientengesprächs und der klinischen und radiologischen Untersuchung festgestellt werden, ob ein Replantationsversuch durchzuführen und Erfolg versprechend ist oder ob eine primäre Stumpfbildung ggf. ein funktionell besseres Ergebnis liefert. Durch ein weitgehend standardisiertes Vorgehen können vor und nach der Operation hohe Erfolgsraten nach Replantationen erreicht werden.

\section{Replantation}

Amputations are relatively rare, but severe injuries for the affected patient. They lead not only to relevant limitations in ability to work and extracurricular activities, but also to a visible physical harm and a loss of sense of integrity. Before the operation begins decisions can already be made as to whether an attempt for replantation is justified or if the patient will benefit from a primary stump formation. Therefore clinical and radiological examinations and a focused patient history are required. Through mainly standardised operative and post-operative procedures, relatively high success rates can be achieved in replantations.

\section{Epidemiologie}

Ca. 5000 Amputationsverletzungen ereignen sich jährlich in Deutschland. Dabei sind die obere Extremität und hierbei insbesondere die Finger mit Abstand am häufigsten betroffen. Am häufigsten ziehen sich Männer zwischen dem 20. und dem 40. Lebensjahr Amputationsverletzungen zu [1].

\section{Definition}

Eine traumatische Amputation ist ein vollständiges Abtrennen von Gliedmaßen ohne Verbindung zum proximalen Stumpf. Das Wiederanpflanzen von Am-

OP-JOURNAL 2015; 31: 86-92

(c) Georg Thieme Verlag KG Stuttgart · New York DOI http://dx.doi.org/10.1055/s-0035-1545972 putaten mit (mikro-)vaskulärer Wiederherstellung der Blutversorgung wird als Replantation bezeichnet. Bei der subtotalen Amputation hingegen bleibt eine Gewebebrücke der Gliedmaße bestehen, während alle relevanten anatomischen Strukturen wie Blutgefäße, Nerven, Sehnen und Knochen durchtrennt sind. Bei subtotalen Amputationen spricht man nicht von Replantation, sondern von Revaskularisation zum Extremitätenerhalt. Je nach Amputationshöhe wird zwischen Makro- und Mikroamputation unterschieden. Die Grenze zur Makroamputation ist nicht ganz scharf definiert. Üblicherweise wird sie an der oberen Extremität im Handgelenk und an der unteren Extremität im oberen Sprunggelenk gesehen. Im DRG-System gilt dagegen die Lisfranc-Gelenklinie als Grenze zur Makroamputation der unteren Extremität, um den erhöhten technischen und mate- riellen Aufwand widerzuspiegeln. Auch an der oberen Extremität ist die komplexeste Höhe die Mittelhandreplantation, sodass diese zu Recht manchmal ebenfalls den Makroreplantationen zugerechnet wird. Während Mikroamputationen der oberen Extremität meist als isolierte Verletzungen auftreten, sind Makroamputationen in ca. $40 \%$ der Fälle mit weiteren Verletzungen assoziiert oder Teil eines Polytraumas.

\section{Präklinik}

Die Grundlagen zu einer erfolgreichen Replantation werden bereits erheblich durch die am Unfallort anwesenden Ersthelfer und den Notarzt gelegt. Der Stumpf wird mittels steriler Kompressen verbunden oder bei spritzenden Blutungen durch einen Druckverband versorgt. Das Amputat wird in trockenen sterilen Kompressen zunächst einzeln in eine sterile Plastiktüte gelegt und verschlossen. Der Amputatbeutel wiederum wird in eine mit kaltem Wasser und wenigen Eiswürfeln gefüllte Plastiktüte so gelegt, wie in Abb. 1 gezeigt. Dabei ist auf eine ausreichende Wassermenge $\mathrm{zu}$ achten, um Erfrierungen der abgetrennten Gliedmaße zu vermeiden. Dies spielt insbesondere eine Rolle bei langwierigen Transportwegen zum nächstgelegenen Replantationszentrum. Bei isolierten Amputationen eines ansonsten stabilen Patienten sollte sofort das nächstgelegene mikrochirurgische Zentrum angesteuert werden, um weitere Verzögerungen zu vermeiden [2]. Im Rahmen einer Mehrfachverletzung wird nach dem Leitsatz „Life before Limb“ die nächstversorgende adäquate Einrichtung anvisiert.

\section{Diagnostik}

Die präoperative Untersuchung sollte, wenn immer möglich, durch den Operateur selbst erfolgen. Dieser kann sich dabei selbst ein Bild vom Verletzungsmuster und der Replantationsfähigkeit sowie vom Allgemeinzustand des Patienten 


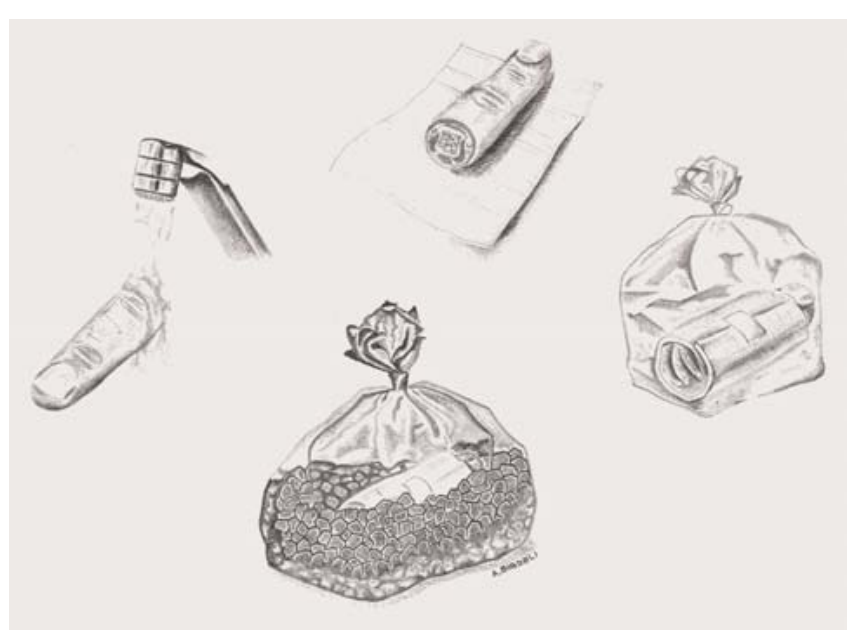

Abb. 1 Schematische Darstellung der korrekten Vorbereitung und des Transports des Amputats in die Klinik.

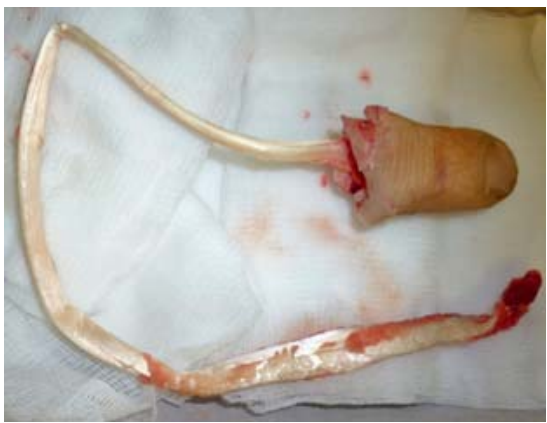

Abb. 2 Quetschverletzung mit Daumenamputation bei proximal ausgerissener Beugesehne.

machen und mit dem Patienten dessen Vorstellungen und Ansprüche erörtern. Die Anamnese sollte neben dem kulturellen Hintergrund private und soziale Aktivitäten (wie z.B. Musiker), v.a. aber auch die beruflichen Erfordernisse einschließen [3]. Wie bei allen offenen oder penetrierenden Verletzungen muss der Status des Tetanusschutzes überprüft und ggf. aufgefrischt werden.

Bei der klinischen Untersuchung kommt der Beurteilung der Weichteilverhältnisse eine wichtige Bedeutung zu. Ideal für eine Replantation sind glattrandige Schnittverletzungen, wogegen Amputationsverletzungen mit Kontusion der Wundränder, wie sie typischerweise nach Kreis- oder Kettensägenverletzungen auftreten, oft nur unter erheblicher Kürzung der Gliedmaße replantiert werden können. Amputationen durch Quetschverletzungen und Ausrissverletzungen haben i.d.R. schlechtere postoperative Ergebnisse, da der intrinsische Schaden des Gewebes typischerweise den sichtbaren Schaden erheblich übersteigt [4]. Operativ sind diese Verletzungsmuster besonders anspruchsvoll in der Versorgung, da es häufig zu einem

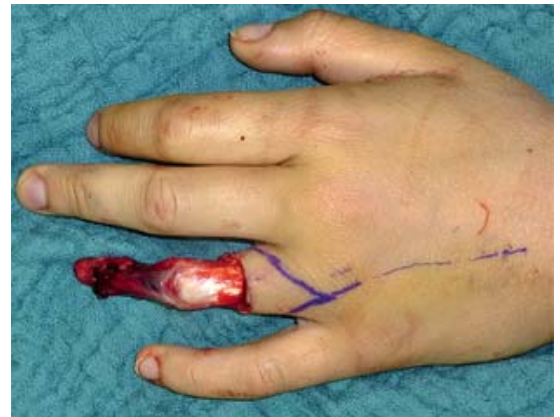

Abb. 3 Ringavulsionsverletzung des linken Ringfingers bei vollständig erhaltenem Sehnenapparat.

Ausreißen der funktionellen Strukturen auf verschiedenen Höhen kommt (Abb. 2). Eine besondere Herausforderung stellen Ringavulsionsverletzungen und hier die Skelettierungsverletzungen dar, bei denen das Weichgewebe vollständig vom intakten Knochen-SehnenApparat abgezogen ist (Abb. 3). Auch bei diesem Verletzungsmuster erleidet das zu replantierende Weichgewebe so ausgeprägte Quetschverletzungen, dass der Replantationsversuch eine deutlich geringere Erfolgsaussicht hat [5].

An präoperativer Bildgebung sollten Röntgenbilder in 2 Ebenen sowohl des Stumpfbereichs als auch der Amputate durchgeführt werden (Abb.4 und 5). Eine Labordiagnostik umfasst mindestens ein kleines Blutbild und den Gerinnungsstatus.

\section{Indikation}

Weiterhin werden klassischerweise absolute und relative Replantationsindikationen unterschieden (siehe Tab.1), auch wenn die relativen Indikationen mit der zunehmenden operativen Sicherheit heutzutage fast immer einen
Tab. 1 Indikationen pro bzw. contra Replantation.

\begin{tabular}{l} 
absolute Indikation \\
- Daumenamputation \\
- Mehrfingeramputation \\
- Mittelhandamputation oder proximal \\
- kindliche Amputationen \\
\hline relative Indikation \\
- Daumen distal des Interphalangeal- \\
gelenks \\
- einzelner Langfinger \\
- Mehretagenschädigung des Amputats \\
Kontraindikation \\
- Wunsch des Patienten zur Stumpf- \\
bildung \\
- Patientenzustand \\
- fehlende Replantationsfähigkeit des \\
Amputats oder des Stumpfes
\end{tabular}

Replantationsversuch zur Folge haben sollten. Distal des Interphalangealgelenks des Daumens gelegene Amputationshöhen stellen eigentlich nur eine relative Replantationsindikation dar. Unserer Meinung nach gilt hierbei jedoch, wenn immer möglich, einen Replantationsversuch zu unternehmen. Es gilt sowohl beim Daumen als auch bei den Langfingern prinzipiell:

Je distaler die Amputationshöhe, desto technisch anspruchsvoller die Replantation, desto besser aber auch die postoperativen Ergebnisse.

Bei der Entscheidung pro oder contra Replantation sollte berücksichtigt werden, dass ein Replantat mitunter einen effizienten Schutz vor Neuromproblemen im Stumpfbereich darstellt.

\section{Operation}

Wenn die Entscheidung zur Replantation getroffen wurde, sollte unverzüglich mit der Operation begonnen werden, um die Ischämiezeit so kurz wie möglich zu halten. In Replantationszentren gelten Replantationen als vitale Indikationen vital für die Amputate, bei Makroamputationen evtl. auch für den Patienten. Die tolerable Ischämiezeit, also die Zeit vom Unfall bis zur erfolgreichen Reperfusion, beträgt bei einer Handamputation 6 Stunden, was bedeutet, dass der Hautschnitt der operativen Versorgung nicht später als 2 Stunden nach Unfall erfolgen sollte. Diese Zeit kann nur bei maximaler Beschleunigung sämtlicher Abläufe eingehalten werden. Auch Fingerreplantationen haben die beste Prog- 


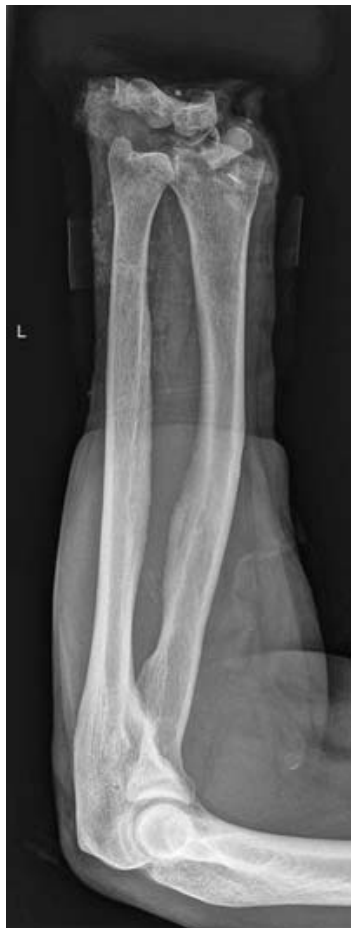

Abb.4 Präoperatives Röntgenbild des Stumpfes nach Amputationsverletzung auf Handgelenkshöhe.

nose bei notfallmäßiger Versorgung. Bei Fingeramputationen können jedoch korrekt gekühlte Amputate noch bis zu 12 Stunden, in Ausnahmefällen sogar bis 24 Stunden nach Unfall replantiert werden. Die in der Vergangenheit gelegentlich geübte Praxis, ein Fingeramputat im Kühlschrank zu lagern und erst am nächsten Morgen im Tagesprogramm zu replantieren, hat in Replantationszentren keinen Platz [6]. Fingerreplantationen folgen i.d.R. einer klar definierten Reihenfolge der zu versorgenden Strukturen, siehe Tab. 2.

Bei Makroreplantationen erfolgt zuerst die Osteosynthese, im Gelenkbereich meist mit Fixateur externe, im Schaftbereich der langen Röhrenknochen durch Plattenosteosynthese nach erheblicher Verkürzungsosteotomie $(4-5 \mathrm{~cm})$. Anschließend werden eine Arterie und eine oder mehrere Venen anastomosiert und die Reperfusion freigegeben. Bei der Makroreplantation ist, ähnlich wie bei der Organtransplantation, darauf zu achten, dass das initial aus dem Replantat zurückfließende Blut nicht in den Kreislauf gelangt. Die Operationsschritte von Replantationen im Einzelnen:

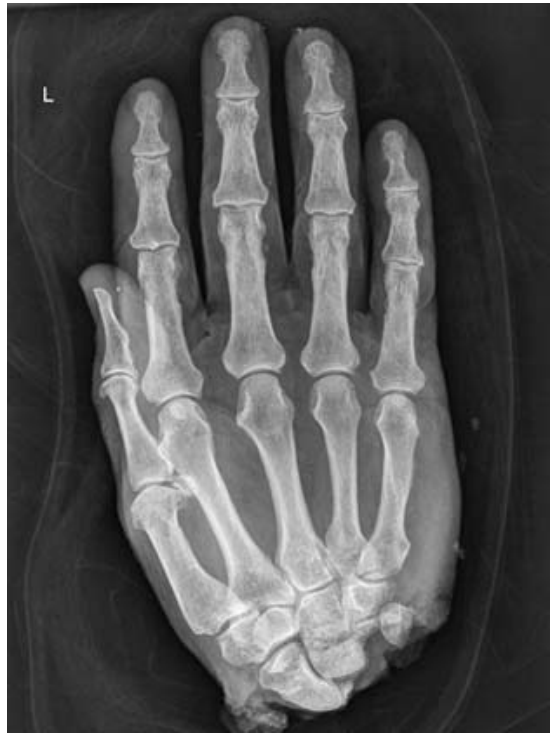

Abb. 5 Präoperatives Röntgenbild des Am-

Tab. 2 Operatives Vorgehen.

1. Aufpräparieren des Amputats (dies kann parallel zu der Narkoseeinleitung am Back-Table erfolgen), Darstellung der Strukturen, Markieren der Nerven und Gefäße

2. Débridement und Aufpräparieren des Stumpfes, Darstellung der Strukturen, Markieren der Nerven und Gefäße

3. Osteosynthese

\begin{tabular}{l}
\hline 4. Naht der Beugesehnen \\
\hline 5. Arterienanastomosen \\
\hline 6. Versorgung der Nervenverletzungen \\
\hline 7. Strecksehnennaht \\
\hline 8. Venennaht \\
\hline
\end{tabular}

9. Weichteilverschluss putats.

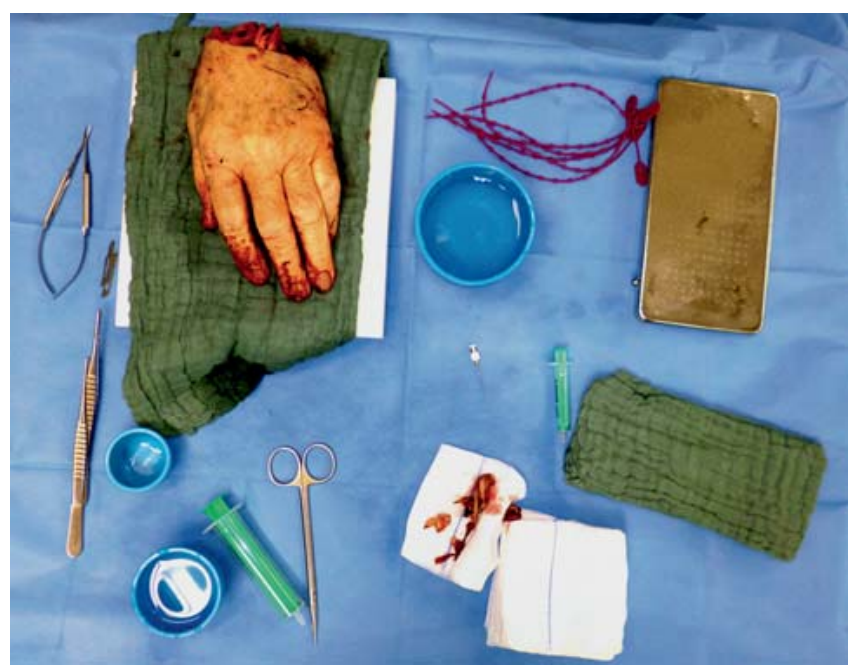

Abb. 6 Beginn der Präparation des Amputats während der Patient eingeleitet wird.

\section{Débridement}

Während der Patient noch anästhesiologisch für die Operation vorbereitet wird, beginnt das Operationsteam bereits mit der Aufpräparation des Amputats (Abb. 6). Dabei werden alle funktionellen Strukturen dargestellt und zur Anastomose bzw. Naht vorbereitet und markiert.

Bei ausgedehnten Knochen- und Weichteilverletzungen kann durch sparsames Kürzen des Knochens die Notwendigkeit von Interponaten für Gefäße und Nerven entfallen [7].

Danach erfolgt die Präparation des Stumpfes unter ausreichender Vergrößerung mittels Lupenbrille oder Operationsmikroskop. Dazu sollte der Arm auf einen Armtisch ausgelagert und bis zum distalen Oberarm abgewaschen werden, um ggf. Gefäßinterponate vom distalen Unterarm, Nerveninterponate oder Spalthaut vom proximalen Unterarm oder Vollhaut aus der Ellenbeuge entnehmen zu können. Bei größeren Defektverletzungen kann auch ein Unterschenkel zur Interponatentnahme steril abgedeckt werden. Generell ist die Anlage einer Oberarmblutsperre zu empfehlen. Um bei einer Mikroreplantation eine zügige Präparation aller funktionellen Strukturen zu erreichen, können sowohl am Stumpf als auch am Amputat longitudinale Inzisionen beidseits in den Mediolaterallinien erfolgen [8]. Insgesamt resultiert bei dieser Technik der Fingerreplantation oft ein $\mathrm{H}$-förmiges Inzisionsmuster. Dieses Inzisionsmuster erlaubt auch im Falle eines Replantatverlusts 


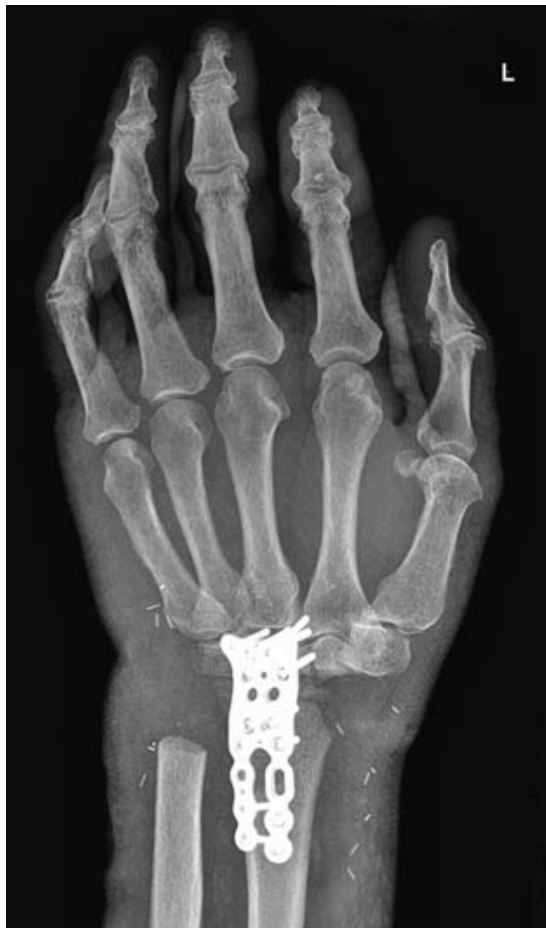

Abb.7 Postoperatives Röntgenbild nach verkürzender Arthrodese bei Handamputationsverletzung (Größe 5).

eine suffiziente Stumpfformung ohne Risiko für Hautnekrosen.

\section{Osteosynthese}

Nach adäquater Vorbereitung des Stumpfes und des Amputats erfolgt zunächst die osteosynthetische Versorgung der Fraktur. In den meisten Fällen einer Mikroreplantation verwenden wir 2 Kirschner-Drähte zur Stabilisierung der Knochen, da somit das Gewebe geschont werden kann. Diese können entweder antegrad oder retrograd eingebracht werden. Das Ziel sollte die übungsstabile Osteosynthese sein, um eine zügige postoperative physiotherapeutische Übungsbehandlung zu ermöglichen. Ansonsten können auch Mini-HPlatten zur Anwendung kommen, welche zwar operativ aufwendiger sind, jedoch eine initial höhere Stabilität ermöglichen. Im Falle von Markoreplantationen können verschiedenste osteosynthetische Verfahren zum Einsatz kommen, wie z.B. Fixateur externe, Schrauben oder Osteosynthese- oder auch Arthrodeseplatten (Abb. 7).

\section{Beugesehnennaht}

Die Beugesehnennaht kann in der sog. "4-Strang-Technik“ mittels Polypropylenfäden der Stärke 3-0 und 4-0 durchgeführt werden. Diese kombiniert als Kernnaht eine Kirchmayr-KesslerNaht 3-0 mit einer U-Naht 4-0 und erlaubt die Beübung von Beugesehnenverletzungen mit frühaktivem Modul. Eine zirkuläre fortlaufende Naht mit einem Polypropylenfaden der Stärke 5-0 bewirkt die feine Adaption der Sehnenstümpfe.

\section{Arterienanastomose}

Grundvoraussetzung zur erfolgreichen Replantation einer Extremität ist die spannungsfreie arterielle Anastomose.

Zur Revaskularisierung einer Extremität kann immer nur eine gut blutfördernde Arterie verwendet werden. Auch muss die Arterie über eine intakte Intima verfügen, um Thrombosen zu vermeiden. Dazu müssen im Zweifelsfall längere Segmente der Arterie reseziert werden. Sollte nach Resektion eine spannungsfreie arterielle Anastomose nicht mehr möglich sein, muss ein Veneninterponat (für Fingerarterien von der distalen Unterarmbeugeseite) in korrekter Flussrichtung eingesetzt werden. Bei Makroreplantationen können proximal gelegene Thromben der großen Arterien (A. radialis und ulnaris oder weiter proximal) in Ausnahmefällen und unter größter Vorsicht mittels Fogarty-Katheter entfernt werden. Niemals jedoch erfolgt eine retrograde Angioplastie im Amputat.

Arterielle Anastomosen an den Fingern werden mit nicht resorbierbaren Nylonfäden der Stärke 9-0 bis 10-0 in Einzelknopfnahttechnik durchgeführt. Prinzipiell sollte immer versucht werden, beide Fingerarterien zu nähen. Sollte dies nicht möglich sein,

gilt als Faustregel, dass immer die näher zum Mittelfinger gelegene Arterie die kaliberstärkere Arterie ist.

Zum Öffnen der Blutsperre nach erfolgter Arteriennaht sollte der systolische Blutdruck des Patienten $110 \mathrm{mmHg}$ nicht unterschreiten. Wenn es trotz sauber durchgeführter Anastomose nicht zu einem Durchfluss und einer Reperfusion des Fingers kommt, kann dies verschiedene Ursachen haben. Ein durch die Manipulation verursachter Vasospasmus kann oft durch kurzes Warten ohne Manipulation, Umspülen mit warmer Ringer-Laktatlösung oder durch externe Applikation von Papaverin (Verdünnung $1: 20)$ behoben werden. Bei ausbleiben- dem Reperfusionserfolg sollte ggf. eine großzügige Resektion der arteriellen Anastomose und erneute Naht unter $\mathrm{Zu}$ hilfenahme eines Veneninterponats erfolgen. Vor Freigabe der Zirkulation werden durch den Anästhesisten 500-2000 Einheiten Heparin i.v. infundiert. Dabei hat sich das aus der Luftfahrt bekannte „read back“ der Medikamente bewährt, d.h. der Anästhesist wiederholt vor der Gabe Medikament und Dosierung, um etwaige Fehler zu vermeiden.

\section{Nervenkoaptation}

Ähnlich der Gefäßanastomose ist für die Wiederherstellung der nervalen Kontinuität die spannungsfreie Nervenkoaptation von höchster Bedeutung. Um herauszufinden, ob eine spannungsfreie Naht möglich ist, und um einen echten Substanzdefekt von der reinen Retraktion des Nervs zu unterscheiden, kann der sog. „stitch test“ durchgeführt werden. Dabei wird eine epineurale Einzelknopfnaht mit einem Nylonfaden der Stärke $10-0$ angelegt. Hält die Naht, so liegt kein Substanzdefekt vor und der Nerv kann mittels weniger weiterer Einzelknopfnähte locker epineural koaptiert werden. Kommt es jedoch zum Ausreißen des Epineuriums, so sollte ein Nerveninterponat vom Unterarm oder Unterschenkel verwendet werden. Der am häufigsten verwendete Spendernerv ist dabei der N. cutaneus antebrachii medialis von der proximalen Unterarmbeugeseite. Dieser liegt oberhalb der Muskelfaszie 1 Fingerbreit medial und 2 Fingerbreit distal des Epicondylus medialis humeri. Prinzipiell können Nervendefekte auch durch ein Nervenkonduit oder Venen-Muskel-Interponate überbrückt werden. Sollte der proximale Nervenstumpf nicht zur Verfügung stehen, kann ggf. eine End-zu-Seit-Transplantation des Nervs erfolgen. Ist jedoch die primäre Rekonstruktion des Nervs nicht möglich, sollte eine frühsekundäre Rekonstruktion erwogen werden.

\section{Strecksehnennaht}

Die Strecksehnennaht an der oberen Extremität erfolgt bevorzugt mit nicht resorbierbarem Fadenmaterial (Polypropylen 4-0) in U-Naht-Technik. Eine fortlaufende zirkuläre Naht ist an den flachen Streckaponeurosen nicht möglich (Abb. 8). 


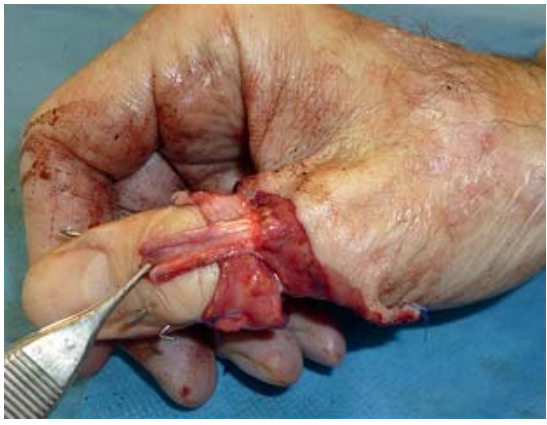

Abb. 8 Vorbereitung zur Naht der Daumenstrecksehne nach vorangegangener Osteosynthese.

\section{Venennaht}

Auch hier gilt die Prämisse der absolut spannungsfreien Anastomose. Sollte dies primär nicht möglich sein, sollten Veneninterponate aus oberflächlichen Venen der distalen Unterarmbeugeseite eingebracht werden. Prinzipiell sollten, wenn möglich 2 Venen pro anastomosierter Arterie genäht werden. Sollten im Ausnahmefall bei Replantationen der Fingerkuppe keine Venen zum Anastomosieren vorgefunden werden, kann auf eine primäre Venennaht verzichtet und ein venöser Abfluss über medizinische Blutegel oder lokale und systemische Heparingabe erzeugt werden, bis sich ein venöses Gefäßnetz gebildet hat [9]. Besteht neben dem venösen Abflussproblem auch ein gleichzeitiger Weichteildefekt, so kann ein venovenöser Lappen eingebracht werden.

\section{Weichteilverschluss}

Nach Blutstillung erfolgt ein lockerer und spannungsfreier Wundverschluss mit Polypropylenfäden der Stärke 4-0 in Einzelknopfnahttechnik. Dabei ist jegliche Kompression auf die Gefäßanastomosen zu vermeiden. Besteht ein Weichteildefekt, der einen primären Wundverschluss nicht zulässt, empfiehlt sich die Spalthautdeckung, die am Finger auch auf anastomosierten Gefäßen möglich ist. Eine temporäre Deckung mit einem Hautersatzmaterial ist auf Gefäßen nicht indiziert und wird nur dann eingesetzt, wenn Hautdefekte z. B. über Sehnen eine sekundäre Weichteildeckung erforderlich machen. Hier ist auf eine regelmäßige Befeuchtung des Gewebes mit Ringer-Lösung zu achten, um ein Austrocknen sicher zu vermeiden (Abb.9). Aufwendige Weichteilrekonstruktionen, wie z. B. lokale oder gar freie Lappenplastiken, werden meist zweizeitig durchgeführt.

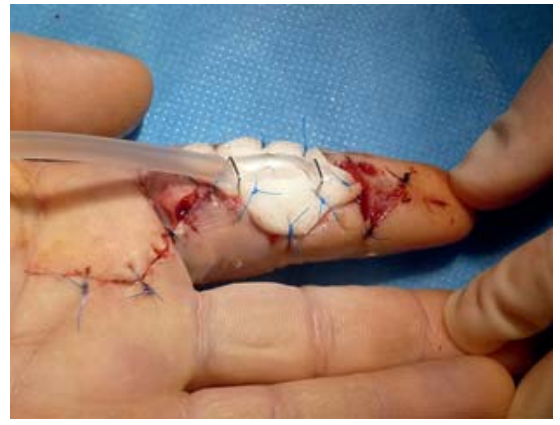

Abb. 9 Coldex-Verband mit Absaugkatheter zum Anfeuchten bei primär nicht verschließbarem Weichteildefekt.

Bei Makroreplantationen ist darauf zu achten, dass die Ischämiezeit der Extremität regelmäßig die Gefahr der Entwicklung eines Kompartmentsyndroms nach sich zieht. Hier erfolgt routinemäßig die vorbeugende vollständige Spaltung aller Faszien sowie die offene Dekompression des N. medianus und des N. ulnaris durch Spaltung des Karpaldachs und der Loge de Guyon. Am Ende der Operation wird ein ausgiebig mit Watte gepolsterter Verband angelegt, der die klinische Beurteilung der replantierten Extremität zulässt (Abb. 10). Der Verband darf niemals zirkulär gewickelt werden, um Kompression zu vermeiden. Eine Gipsruhigstellung ist bei Replantationen und Revaskularisationen in der initial postoperativen Phase aufgrund des Risikos der venösen Stauung nicht empfehlenswert.

\section{Postoperative Versorgung}

Neben der adäquaten Analgesie, die regelhaft NSAIDs und Opioide umfasst, kommt anfangs der Antikoagulation eine besondere Bedeutung zu. In vielen Kliniken erfolgt eine initiale therapeutische Antikoagulation mittels Clexane-Spritzen oder Heparinperfusor, welche im späteren Verlauf auf eine prophylaktische Antikoagulation reduziert werden kann. Zusätzlich erhält der Patient häufig für 2 Wochen postoperativ $100 \mathrm{mg}$ Aspirin oral. Eine mehrtägige postoperative Antibiotikaprophylaxe mit Cefuroxim oder Clindamycin bei Penicillinallergie senkt das Risiko von Infekten in den anfangs geringer perfundierten bzw. suboptimal ernährten Geweben wie Knochen oder Sehnen. Idealerweise wurde bereits intraoperativ ein Plexuskatheter gelegt. Dieser dient gleichermaßen der Gefäßerweiterung durch Sympathikolyse und der schmerzfreien postoperativen Beübung. Die Lagerung der Extremität erfolgt auf Herzhöhe. Fünf Tage Bettruhe

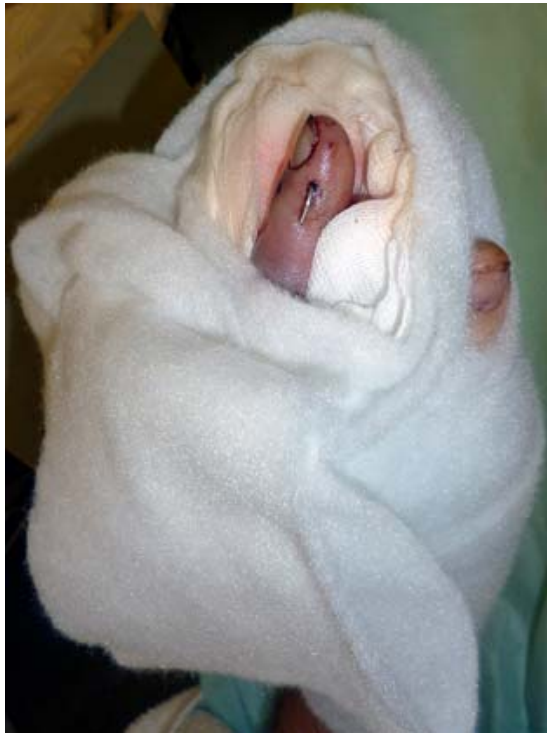

Abb. 10 Verband nach Replantation.

sind sinnvoll, um eine venöse Stauung zu vermeiden. In den ersten 7 Tagen erfolgen anfangs stündliche Durchblutungskontrollen des Replantats anhand der Hautfarbe, der Rekapillarisierung, des Hautturgors sowie der Wärme des Replantats. Bei Makroreplantationen sind Sauerstoffclips ein häufig verwendetes Mittel, um die Durchblutung der replantierten Extremität zu testen. Wegen der Asensibilität besteht jedoch die Gefahr von Drucknekrosen, weshalb aufklebbare weiche Clips alle 2 Stunden umgesetzt oder starre Clips nur stündlich zur intermittierenden Messung eingesetzt werden. Im späteren Heilverlauf ist dem Patienten eine Nikotinkarenz von mindestens 12 Wochen unbedingt anzuraten [10].

Am 2. postoperativen Tag wird mit der Physiotherapie begonnen, die anfangs in vorsichtigen, aktiv geführten Bewegungen besteht. Von Anfang an sollten auch die angrenzenden Finger vorsichtig durchbewegt werden, um eine angstbedingte Schonhaltung und daraus resultierende Funktionseinschränkungen der Nachbarfinger zu vermeiden. Bei Makroreplantationen wird außerdem etwa 7-10Tage nach Operation mit einem Funktionstraining der unverletzten Hand („Einhändertraining“) begonnen, um die Selbstständigkeit im Alltag noch in der Heilungsphase zu gewährleisten.

Postoperative Röntgenaufnahmen können durch Transport des Patienten und Lagerung der Hand die Replantation gefährden und sind deshalb in den ersten 


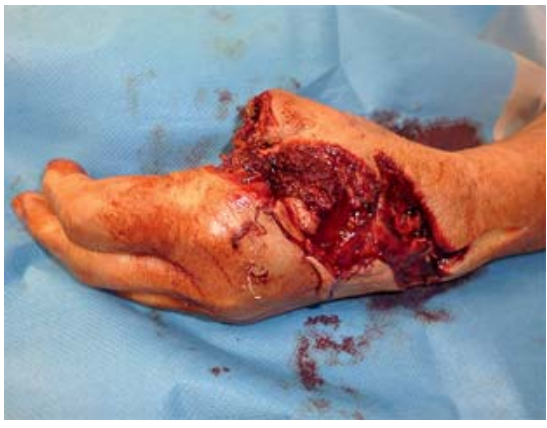

Abb. 11 Präoperative Aufnahme bei Daumenamputation mit ausgeprägtem streckseitigem Weichteilschaden.

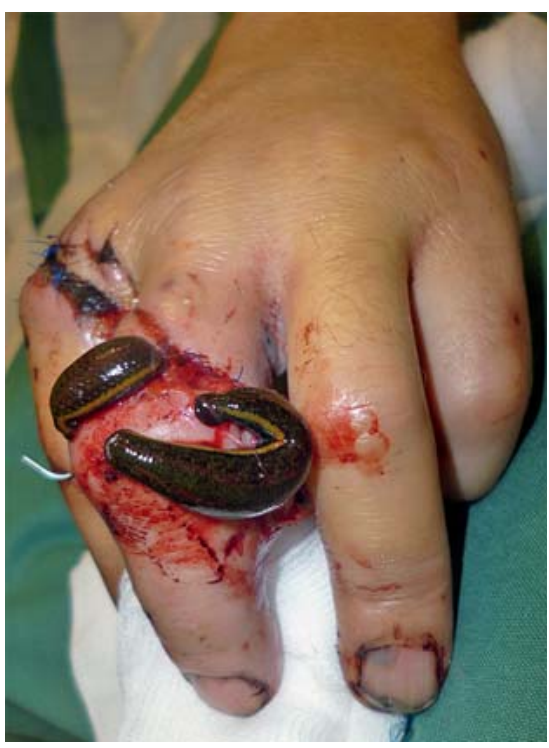

Abb.13 Medizinische Blutegel auf einem Replantat mit venösem Abflussproblem.

7 Tagen nur im Ausnahmefall erforderlich. Die knöcherne Konsolidierung nimmt oft mehr Zeit in Anspruch, da die Perfusion des Knochens auch bei erfolgreicher Replantation nicht immer von Anfang an optimal ist. Die knöcherne Konsolidierung von Fingerfrakturen ist also manchmal erst nach 8 statt nach 6 Wochen gegeben, sodass auch erst dann ein zunehmender Belastungsaufbau möglich ist (Abb. 11 und 12).

\section{Komplikationen}

In den ersten 7 Tagen nach Replantation ist die Thrombosegefahr der anastomosierten Gefäße am höchsten. Sollte es zu einer arteriellen Minderperfusion kommen (weißes Hautkolorit, verlangsamte Rekapillarisierung, schlaffer Turgor der Fingerbeere) kann ggf. die Extremität etwas tiefer gelagert, der Verband geöffnet und zum Wärmen in Goldfolie einge-

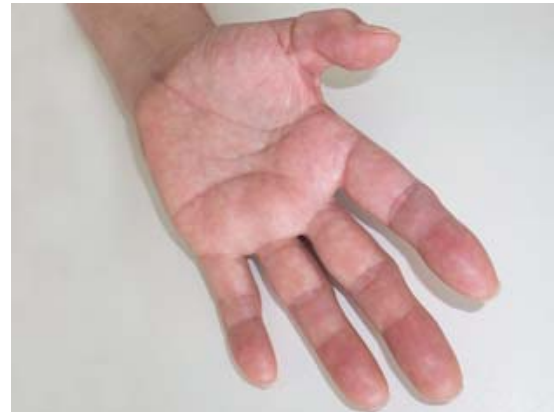

Abb. 12 Postoperatives Ergebnis nach vollständiger Konsolidierung.

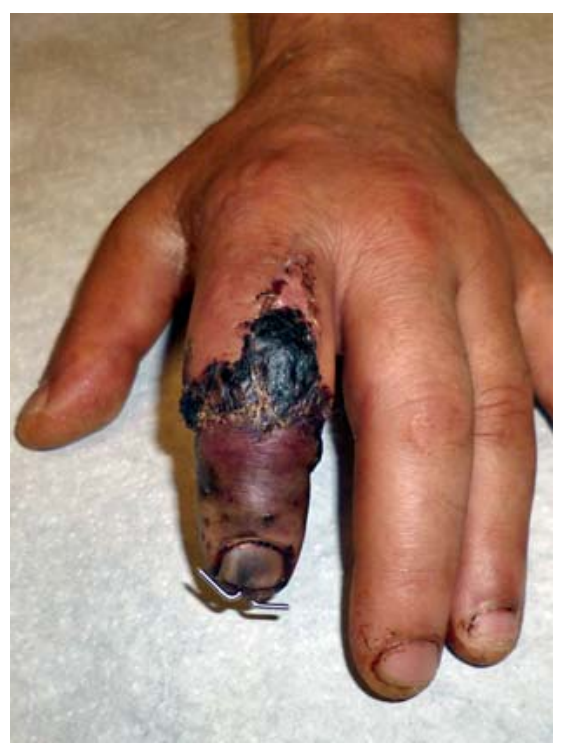

Abb. 14 Livide Verfärbung nach frustranem Replantationsversuch des linken Zeigefingers.

packt werden. In der Regel aber muss bei Verdacht auf einen arteriellen Thrombus großzügig die Indikation zur Revisionsoperation gestellt werden, vor allem innerhalb der ersten 3 Tage nach Replantation. Sollte sich intraoperativ ein Gefäßverschluss zeigen, so wird dieser vollständig reseziert und meistens mittels Gefäßinterponat überbrückt. Gelegentlich und bei früher Revision genügen schon das Öffnen einer einschnürenden Hautnaht und eine sekundäre Spalthauttransplantation.

Bei venöser Stauung der Extremität (livide Verfärbung, schnellere Rekapillarisierung, pralle Pulpa) gibt es neben dem Hochlagern, dem Öffnen des Verbands und der Revisionsoperation noch die Möglichkeit des Einsatzes von medizinischen Blutegeln, vor allem ab dem 5. Tag nach Replantation (Abb. 13). Durch das von den Egeln freigesetzte Hirudin ist es

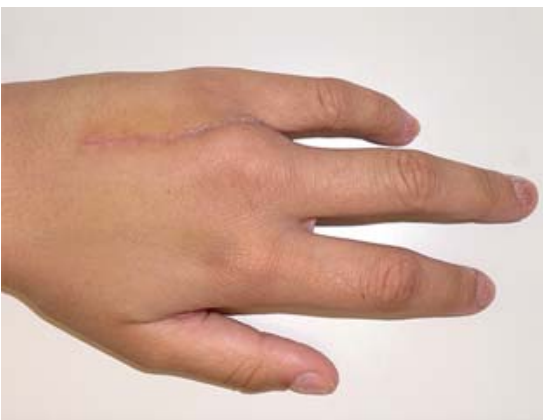

Abb. 15 Handverschmälerung nach Amputationsverletzung des Ringfingers.

möglich, auch bei komplettem venösem Verschluss durch eine kontrollierte venöse Blutung das Replantat über die Zeit bis zur Neubildung eines dermalen Venennetzwerks zu retten, das sich meist nach 7-8 Tagen ausgebildet hat. Zu beachten sind regelmäßige Blutbildkontrollen und das Umstellen der Antibiose auf Ciprofloxacin, welches gegen die von Egeln übertragenen Aeromonas-hydrophila-Infektionen wirkt.

Sollte der Replantationsversuch in der Operation oder im postoperativen Verlauf scheitern, so ist eine gute Patientenführung von großer Bedeutung (Abb. 14). Während zu Beginn eine Unterstützung der Verlustbewältigung im Vordergrund steht, rückt im Verlauf die Physiotherapie, die Planung von möglichen sekundären Rekonstruktions-, form- und funktionsverbessernden Eingriffen (z.B. Verschmälerungsoperationen) oder auch die Prothesenversorgung in den Mittelpunkt (Abb. 15). Hierbei sollte der Patient einen kompetenten Partner in der behandelnden Klinik finden, der über die rein operative Versorgung hinaus den Patienten im Heilungsverlauf begleitet.

\section{Literatur}

${ }^{1}$ Towfigh $H$, Hierner $R$, Langer $M$, Friedel $R$. Handchirurgie. 1.Aufl. Berlin, Heidelberg: Springer; 2011

${ }^{2}$ Haas EM, Volkmer E, Holzbach $T$ et al. Über Versorgungsstrukturen und Möglichkeiten der Optimierung durch Vernetzung bei schweren Handverletzungen und Replantationen. Handchir Mikrochir Plast Chir 2013; 45: 318-322

3 Gonser P, Medved F, Schaller HE et al. Ökonomischer Profit von Fingerverletzungen in einem handchirurgischen Zentrum - Replantation vs. Amputation. Handchir Mikrochir Plast Chir 2013; 45: 350-353

${ }^{4}$ Sebastin SJ, Chung KC. A systematic review of the outcomes of replantation of distal digital amputation. Plast Reconstr Surg 2011; 128 : 723-737 
5 Sears ED, Chung KC. Replantation of finger avulsion injuries: a systematic review of survival and functional outcomes. J Hand Surg Am 2011; 36: 686-694

${ }^{6}$ Lin CH, Aydyn N, Lin YT et al. Hand and finger replantation after protracted ischemia (more than 24 hours). Ann Plast Surg 2010; 64: 286-290

${ }^{7}$ Barbary S, Dap F, Dautel G. Finger replantation: surgical technique and indications. Chir Main 2013; 32: 363-372
${ }^{8}$ Wolfe SW, Pederson WC, Hotchkiss RN, Kozin SH. Green's Operative Hand Surgery. 6th ed. London: Churchill Livingstone; 2011

${ }^{9}$ Beier JP, Horch RE, Kneser U. Chemical leeches for successful two-finger re-plantation in a 71-year-old patient. J Plast Reconstr Aesthet Surg 2010; 63: e107-e108

${ }^{10}$ Prucz RB, Friedrich JB. Upper extremity replantation: current concepts. Plast Reconstr Surg 2014; 133: 333-342
Dr. med. Nico Leibig

Assistenzarzt

Dr. med. Berthold Bickert

Leitender Oberarzt

Leiter der Sektion Handchirurgie

Prof. Dr. med. Ulrich Kneser

Direktor der Klinik

Klinik für Hand-, Plastische und

Rekonstruktive Chirurgie,

Schwerbrandverletztenzentrum

Berufsgenossenschaftliche

Unfallklinik Ludwigshafen

Ludwig-Guttmann-Straße 13

67071 Ludwigshafen

nico.leibig@bgu-ludwigshafen.de 